\title{
SocArXiv
}

Preprint : January 22, 2021

doi.org/10.31235/osf.io/u4zcs

\section{TSINC: A Plagiarism-resistant Online Testing Method}

\author{
Yingwei Wang \\ University of Prince Edward Island, Canada
}

\begin{abstract}
TSINC is a new online testing method. The acronym TSINC represents its five characteristics: Time-pressed, Sequential, Individualized, Not searchable, and Calibrationbound. Its primary feature is that it can be used to organize plagiarism-resistant online testing without human monitoring. In this paper, the logical basis of this method, its implementation details, applicable areas and limitations, test cases, and so on are reported.
\end{abstract}

Keywords: evaluation method, testing method, online testing, exam, plagiarism, fraud, academic misconduct, impersonation, cheating, remote education, teaching, education.

\section{Introduction}

The widespread of the COVID-19 pandemic forced most universities and schools to turn to online teaching. While video conference platforms can support online lecturing, online testing became the most challenging component for many instructors. The traditional testing methods are based on human proctoring, but proctoring is impossible in an online testing environment.

To maintain test fairness, some instructors required test participants to turn on their cameras; some instructors reduced the test weights in the course; some instructors canceled the tests and used term essays to evaluate participants. These measures are not ideal; they cannot provide a plagiarism-resistant testing method to evaluate test participants.

In this paper, a new online testing method, TSINC, is introduced. The TSINC testing method has the following features:

- It can evaluate test participants fairly and meaningfully without human proctoring or any other form of human monitoring.

- It cannot eliminate plagiarism or other forms of academic misconduct completely; however, it can lower the grades of plagiarists and make plagiarism less attractive. 
- It can be used in most universities and schools.

- It can be implemented easily in most Learning Management Software (LMS), such as Moodle and Google Classroom.

The rest of the paper is organized in the following way: Section 2 discusses the underlying ideas and logical foundations of this new method; Section 3 describes the details of the TSINC testing method; Section 4 clarifies a few key issues related to this method; Section 5 reports some cases of TSINC tests; Section 6 provides the conclusions.

\section{The Logical Basis of Plagiarism-resistant Online Testing}

According to the Oxford Dictionary, Plagiarism is "The practice of taking someone else's work or ideas and passing them off as one's own." In practical words, plagiarism is an act of fraud or academic misconduct. In an online testing environment, the major forms of plagiarism include (1) communicating with someone else to exchange answers or related knowledge; (2) searching online to obtain related knowledge; (3) searching other resources, such as course notes/books, to obtain related knowledge.

Admittedly, it is impossible to eliminate plagiarism in an online testing environment. When a test participant commits plagiarism, the instructor/test organizer could not know that such plagiarism is happening and could not identify which answers were the results of plagiarism.

\subsection{Making Time Matter}

Plagiarism cannot be eliminated completely, but can something be done about these activities? Our first response was that we could make plagiarism more difficult, hoping that such activities would be reduced.

If the test papers are identical for the class, the copying process would be easy. If each test participant faces different test questions, plagiarists have to verify that the copied answers are in the right places. Currently, it is easy to randomize question orders and to variate each question. Once the questions in a test have been randomized and variated, plagiarism would be more difficult. Section 3.3 discusses related details.

Instructors could also form questions in such a way that the answers cannot be obtained directly from books, course notes, or a simple online search. Plagiarists would need to think and reason to obtain the correct answers. In this way, the difficulty of plagiarism is increased. Section 3.4 discusses related details.

Such approaches have been used by many instructors, but they cannot go far. Even though plagiarism was reduced, plagiarists could still benefit from such activities. These approaches are not ideal solutions. For example, a plagiarist's grade might be $40 \%$ if he/she did not plagiarize; if he/she committed plagiarism, his/her grade could be $60 \%$.

Our goal is not to find a testing method that only reduces plagiarism. Instead, our goal is to find a plagiarism-resistant testing method that makes these activities useless. For example, a test participant's grade could be $40 \%$ if he/she did not commit plagiarism; if he/she plagiarized, his/her grade could be only $35 \%$.

How do we reach this goal? 
Let us examine plagiarists' situation in traditional tests. Having answered a small number of questions that they know, they still have abundant time. They can either submit their test paper early or plagiarize. Whatever grade they obtain through plagiarizing would be their net gain.

Supposing the instructor reduce the test's time limit and let the potential plagiarists have barely enough time to answer the small portion of questions they know. Can they still commit plagiarism? No, they cannot.

We realized that time is the key to control plagiarism. Plagiarists have to pay a special cost in these activities: a time cost. However, the time cost is only worth discussing when the time limit of a test is reduced significantly. In traditional tests, time is abundant.

Combating plagiarism goes in two directions. The first one is to increase the time needed for a plagiarist's activities to be successful; the second one is to reduce the tests' time limit. Randomizing question order and making questions not searchable can be used in the first direction. They can not only increase the difficulty of plagiarism, as discussed above, but also increase the time needed for plagiarism to be successful. They are useful approaches and should be used in the new testing method. Details about randomizing question order and making questions not searchable are discussed in Section 3.3 and Section 3.4. Here we concentrate on the second direction.

Assuming that questions are composed such that when a test participant reads each question, he/she either knows the related knowledge/skill or does not know. For these questions, an honest learner would need much less time than a plagiarist would need.

Once we realized the difference in required time between honest learners and plagiarists, the idea of reducing test time came up. Reducing test time is the first step in creating a plagiarism-resistant testing method. If a test participant commits plagiarism, he/she needs to talk to people, verify questions, turn book pages, go over notes, and search online. On the contrary, honest learners do not need such extra time because they only need to put in the answers they know. This time difference ensures that plagiarists will answer fewer questions than honest learners will in the same time. Plagiarists will be in an unfavorable position as they answer the remaining questions; what they have gained through plagiarism will be lost.

For example, all students have to answer 50 questions in 30 minutes. If a plagiarist spends 15 minutes looking for answers everywhere and successfully obtains correct answers for 10 questions, it is unlikely that he/she will do well in solving the remaining 40 questions within 15 minutes. We expect that this offender will perform badly in the whole test.

Reducing the sitting time of tests makes a plagiarism-resistant testing method possible. Nevertheless, it also brings in something undesirable. Many test participants, including honest learners, may not be able to answer all the questions correctly in a short time. The following situation may occur: a test participant may obtain $95 \%$ if enough time is given, but he/she only obtains $70 \%$ because there has not been enough time to consider and solve all questions. The approach of reducing the time limit, randomizing question order, and forming notsearchable questions, establishes a discriminatory effect on plagiarists. To plagiarize, offenders need to spend time on their activities; however, honest test participants do not have such time cost because they do not perform these activities. When the test time is short, discrimination in time would be converted into discrimination in grades.

What we have achieved through the above approach is valuable. Although this approach causes a new problem in that all test participants obtain lower grades due to lack of time, its 
benefits are larger. Additionally, this new problem can be solved by grade calibration. The details of the calibration process are discussed in Section 3.5.

Making the cost of time in testing significant and increasing the time cost of plagiarism are the core ideas of the proposed plagiarism-resistant online testing method.

\subsection{Making Time Matter More}

To strengthen the new approach's potential, we would like to incorporate another mechanism: sequential question answering.

Sequential question answering means that each question is presented on a separate page. A test participant must progress through the questions in order; he/she cannot return to previous pages nor skip ahead. This mechanism is supported by many LMS platforms, but it has not been widely used. The unpopularity of sequential question answering could be related to two issues. The first issue is that it contradicts the norms in traditional testing methods, whereby test participants should be able to navigate through all the questions freely; the second issue is that its significant advantages are unknown.

In the context of developing a plagiarism-resistant online testing method without human monitoring, sequential question answering demonstrates its power and significant advantages. As illustrated in Section 2.1, reducing the time of online tests is the first step in creating a plagiarism-resistant testing method. In the same way, incorporating the sequential characteristic would be the second step.

First, let us analyze this characteristic at the level of each question. When sequential question answering is used in a time-pressed test, a potential plagiarist must make a choice when he/she does not know how to solve a specific question:

- Choice 1 and consequences: (1) start plagiarism immediately; (2) extended time would be spent on talking to other people or searching online/notes/books; (3) the correct answer might be obtained; (4) the time spent would hurt the overall grade.

- Choice 2 and consequences: (1) leave this question empty or make a guess; (2) let this question pass, and the grade of this question likely is lost; (3) if the correct answer is available later through plagiarism, it cannot be filled in.

Either choice will lead to a low grade. This result is fair because he/she does not know how to solve this question.

Then, let us analyze this characteristic at the level of the whole test. Anyone who wants to answer all the questions in a TSINC test must distribute the time almost evenly; only a very short time can be dedicated to a specific question. For an honest learner, he/she prepared related knowledge/skill; his/her task is to solve questions using such knowledge/skill. A plagiarist must ask other people, search online, or turn pages in a book to find something. All these activities may fail. The successful attempts must be completed within the short, dedicated time periods; otherwise, they are useless. It is reasonable to say that plagiarists face much bigger pressure than honest learners do. Plagiarists may have success in some questions, but overall, they are punished for the plagiarism they committed.

The sequential question answering characteristic is powerful, but this characteristic itself will not enable an effective plagiarism-resistant feature if it is not combined with the time-pressed 
characteristic. When enough time is provided, plagiarists would still be able to answer many questions through plagiarism. Other characteristics discussed in Section 3.3 and Section 3.4 are also necessary to realize the benefits of sequential question answering.

\section{The TSINC Testing Method}

The TSINC testing method has five characteristics: Time-pressed, Sequential, Individualized, Not searchable, and Calibration-bound. We introduce the details of these characteristics in the following:

\subsection{Time-pressed}

A TSINC test should be time-pressed; time-pressed is the key characteristic of a successful TSINC test. An ideal TSINC test should make everyone busy; no one has spare time.

- A TSINC test should keep honest learners busy. The honest learners are the ones who might be asked to share answers with other test participants; they are the passive source of plagiarism. When they are busy, they do not have time to help others. They also have a good excuse to refuse such requests: I am busy. I need to finish my own work.

- A TSINC test should keep potential plagiarists busy. These people are the active source of plagiarism. When the test is time-pressed for honest learners, it will not be easy for potential plagiarists. Yes, they can commit plagiarism to answer some questions, but plagiarism will cost their limited time and lead to a low grade overall in the test.

It is important to introduce this characteristic to test participants before the tests and explain the reasons. Otherwise, test participants will be surprised and frustrated. Instructors should clearly explain to the test participants that an adjustment or calibration process will be performed after the test. Thus, the grade of the test may not be the final grade of the test. With these explanations, test participants would understand that the goal to take a TSINC test is not to answer every question correctly; instead, the goal should be to reach the best overall performance. Each test participant should develop his/her time management strategy.

\subsection{Sequential}

The importance of sequential question answering has been discussed in Section 2.2. Here is the analysis of some details.

If a test participant figures out the correct answer when the question has already passed, the sequential characteristic blocks the answer to be filled in the test. Such blocking is applied to every test participant, regardless if it is a plagiarist or an honest learner. Does this blocking have the same influence on these two kinds of test participants?

We know that the questions are designed such that when a test participant reads the question, he/she either knows the related knowledge/skill or does not know. Honest learners rely on their knowledge to answer questions. It is possible that he/she could not figure out the answer immediately but figures it out later; the frequency of that occurring would be quite low.

Plagiarists do not know the related knowledge/skill; they rely on various sources to obtain answers. It is more likely that they "figure out" the correct answers later. 
For example: in a TSINC test, an honest learner may be blocked once because he/she realizes that an answer he/she uses before is wrong; a plagiarist may be blocked five times because he/she "figures out" many answers that he/she misses previously. Plagiarists might get the answers somehow, but they may not be able to control the time that the answers are available. The sequential characteristic has different influences on honest learners and plagiarists. Such discrimination also contributes to the plagiarism-resistant feature of the TSINC testing method.

For the sequential characteristic to be kept meaningful, the number of questions in a TSINC test should be large enough. A TSINC test should have at least 20 questions. In more preferable cases, a TSINC test should have 30 or more questions.

With a TSINC test in place, plagiarists will not put in vain efforts. Thus, they may give up plagiarism, which is what instructors want.

\subsection{Individualized}

When an instructor prepares a TSINC test, two steps are involved. The first step is to devise some question groups. Each group has one or more questions; all the questions in one group are very similar. Questions in one group could be generated from one question by variating the numbers/parameters in the question.

In the second step, the order of groups should be generated randomly for each test participant, with everyone's test having a different order. For each group, only one question is chosen randomly from inside the group and is presented in the test.

The question set presented to each test participant is essentially the same for all participants, but the order of questions is different for everyone. A's Question 1 could be B's Question 15. Additionally, A's Question 1 could be slightly different from B's Question 15 in numbers or other minor factors; the correct answer for A's Question 1 could be 10, but the correct answer for B's Question 15 could be 20 .

To make things more complicated, even if the correct answer for A's Question 1 and the correct answer for B's Question 15 are both 10, if this is a multiple-choice question, the correct choice for A's Question 1 could be A, but the correct choice for B's Question 15 could be $\mathrm{C}$.

In such individualized tests, copying answers could be quite time-consuming. The individualized characteristic contributes greatly to the plagiarism-resistant feature of the TSINC testing method.

The individualized characteristic is available in many LMS systems, and some instructors already use them in their tests. But this characteristic alone will not enable an effective plagiarism-resistant feature if it is not combined with other characteristics. If the test is not time-pressed and sequential, plagiarists would still be able to successfully perform plagiarism.

\subsection{Not searchable}

When an instructor prepares questions for a TSINC test, he/she should ensure that the answers cannot be obtained by a simple online search or a course-note skimming. For instance, questions such as "What is (a concept or an acronym)?" or "Why is (something) not allowed?" should not be used. 
While searching online or searching in books/notes would help to answer questions, the questions should be made in such a way that searching cannot provide answers directly; instead, answers can only be obtained with test participants' thinking and reasoning based on the search results.

This characteristic sets a high standard for instructors. Instructors have to design test questions carefully; otherwise, plagiarism will still occur.

This characteristic is desirable, but on its own, it will not enable an effective plagiarismresistant feature if it is not combined with other characteristics. If the test is not time-pressed and sequential, plagiarists can still obtain answers from other test participants.

\subsection{Calibration-bound}

The above four characteristics exert a negative impact on plagiarism and make TSINC tests plagiarism-resistant; however, these characteristics also bring in new issues. In TSINC tests, the time-pressed and the sequential characteristics negatively influence the grades of the whole class. In other words, TSINC tests are always too difficult. This factor must be compensated so that a planned adjustment or calibration process is performed after each test.

The grade adjustment process gives people the impression that the grades in TSINC tests are arbitrarily set; therefore, TSINC is not a fair evaluation method.

Such a grade adjustment process also exists in traditional testing methods. If an instructor finds out that the grades shift to the low end for the entire class because the test questions were too difficult, he/she may decide to increase the grades for the whole class based on a mapping function. If an instructor finds out that the grades shift to the high end for the whole class because the test questions were too easy, he/she may decide to decrease the grades for the class based on a mapping function. There is a special term used in these situations: to curve the grade.

In practice, instructors usually observe test results through a statistic, for example, the mean of all the grades, or a group of statistics, for example, the mean and median of all the grades. If a statistic shifts away from the instructor's expected value, he/she may adjust the grades.

Is there an expected statistic value for a test? It is possible that an instructor cannot point out the specific amount of the expected value, but he/she knows which value is right and which value is wrong.

Instructors may find in certain cases that the test statistic shifts to one end. If the shifting is significant, the instructor has to curve the grade; if the shifting is minor, the instructor may leave the grades as they are, and test participants will have to accept the results. Overall, grade adjustment to the entire class is performed rarely on traditional tests. It is considered a remedial measure.

TSINC tests also have grade shifting. Compared to traditional testing methods, TSINC tests' grade shifting has the following characteristics: (1) the shifting is always toward the low end of grades; in other words, TSINC tests are always too difficult. (2) the shifting exists in every TSINC test; (3) the shifting amount is significant in most cases.

Due to the above characteristics, grade adjustment is mandatory and an integral part of TSINC tests. From this perspective, a TSINC test could be fairer than a traditional test: in a traditional test, the instructor may choose not to curve grades when he/she thinks the shifting was a minor one. 
Because grade adjustment or curving must take place in every TSINC test or is bound to happen, we need to investigate the methods of performing such an adjustment. The term calibration replaces adjustment from now on to emphasize that this process is based on objective facts and is not arbitrary.

Many calibration methods can be developed depending on the goals and the character of the test. Here are only two calibration methods:

\section{Target-guided Calibration Method}

The target-guided calibration method includes the following steps:

1. Before a TSINC test is held, the instructor may choose a mapping function and an expected statistic value.

2. After the TSNIC test, if the statistic value is different from the expected statistic value, the mapping function will be applied with proper parameters.

The following mapping functions are just a few examples:

$$
\begin{gathered}
\mathrm{y}=10 \sqrt{x} \\
\mathrm{y}= \begin{cases}x+k & \text { if } \quad x+k \leq 100 \\
100 & \text { if } \quad x+k>100\end{cases} \\
\mathrm{y}=\frac{x^{2}}{100} \\
\mathrm{y}=x+k_{1}+k_{2}-\frac{k_{2}\left(x+k_{1}\right)}{100}
\end{gathered}
$$

The above mapping functions were devised for different purposes. I believe that mapping function (4), when parameters $k_{1}$ and $k_{2}$ are chosen properly, is the best fit for TSINC tests. For example, if $k_{1}=10$ and $k_{2}=5,80 \%$ will be mapped to $90.5 \%$; $60 \%$ will be mapped to $71.5 \%$; $50 \%$ will be mapped to $62 \%$. The parameters $k_{1}$ and $k_{2}$ determine the applicable range of the mapping function.

The following example demonstrates the Target-guided Calibration Method. Before a TSINC test is given, the instructor chooses a mapping function, say, mapping function (4), and chooses the target mean of the whole class, say, $70 \%$. After the test, the parameters $k_{1}$ and $k_{2}$ will be determined accordingly, and the mapping function will be used to calibrate the grades of the whole class.

In this procedure, the instructor does not need to make a subjective decision and will not be criticized for manipulating grades arbitrarily.

The calibration process could compensate for the influence of the time-pressed and the sequential characteristics. For example, suppose a test participant obtained $65 \%$ in a test due to time limit and sequential question answering. If this test participant is among the top $5 \%$ of the class, the grade he/she finally obtained in the test could be $90-95 \%$ after the calibration process. 


\section{Accurate Mapping Calibration Method}

For the accurate mapping calibration method to be used, there should also be an accompanying traditional test together with the TSINC test. These two tests should have the same sets of questions. A few chosen test participants, as a representative sample of all test participants, should attend both tests. They should take the traditional test first with proctoring and then take the TSINC test.

The target is to calibrate the TSINC test grades using a consistent mapping function, and for the test participants of both tests, their calibrated TSNIC test grades should be the same or close to their traditional test grades.

This calibration method is more complicated, and it may not be practical if all the tests are online. It is likely that a test participant's second grade is higher than the grade if the questions were presented to him/her for the first time. Thus, the accurate calibration method may not be accurate.

\section{Discussions}

\subsection{Impersonation}

The TSINC testing method is plagiarism-resistant, but it is not resistant to all academic misconducts. Specifically, a TSINC test participant can still commit impersonation, i.e., someone else pretends to be the test participant. Impersonation, either in person or electronically, is a more serious academic offense than plagiarism. In many universities, the recommended sanction is expulsion. Impersonation is rare than plagiarism.

Online impersonation can be dealt with by other technologies, such as various biometric technologies. Here we briefly discuss the conditions that may reduce the possibility of impersonation.

To impersonate someone in a test, the impersonator needs to comprehend the knowledge/skills needed in the test. If the knowledge/skills are widely available, the instructor should be aware of the possibility of impersonation. However, if the knowledge/skills are not widely known, impersonation is less likely to happen.

Most university courses and training programs cover special knowledge and skills. Courses vary in coverage, focus, and style when taught by different instructors at different universities. For a course that is updated often, a student who has taken this course before may not do well definitely in a new test; this course is impersonation-safe. For a course that is rarely updated, impersonation could be a larger issue.

All students in elementary and high schools have classes at the same time. Impersonation is less likely to happen if tests are given at school time, even though online tests can be held at any time.

\subsection{TSINC's Applicable Areas}

If the time limit is set correctly, a TSINC test can prevent most answer copying among test participants. In other words, searching online/books/notes is the only possible form of plagiarism in a TSINC test. 
The following simple criteria may help to determine if the TSINC testing method is applicable: if an honest participant needs less time to answer the questions than a plagiarist needs to obtain the answer through searching online/books/notes, the TSINC testing method is applicable to these questions; otherwise, it is not applicable.

The most suitable questions are such that when a test participant reads each question, he/she either knows the related knowledge/skill or does not know. For these questions, an honest learner would need much less time than a plagiarist would need.

TSINC tests can be used not only on those questions that prompt immediate answers but also in areas where it takes a long time to answer a question, such as mathematics or arts, because (1) the questions are not searchable because they are made with such goals in mind; (2) the number of questions are quite large, so that answer copying is almost not possible. The challenge is that whether many not-searchable questions can be made in these areas.

\subsection{Online Closed-book Test}

In an online testing environment, instructors are forced to give open tests. Test participants are permitted to talk to each other, to search online/books/notes. Many instructors are reluctant to give open tests because many such open tests are meaningless, but they have no choice because monitoring is impossible. Using the TSINC testing method, instructors can give closed-book tests online. Nevertheless, instructors announce that the tests are not open tests, that test participants cannot exchange information or have discussions, and that they are not allowed to use the Internet, books, course notes, or other resources during the test. Even though these rules are difficult to enforce physically in an online environment, they indicate that the knowledge/skill involved should be mastered independently. The TSINC testing method reintroduces closed-book tests and produces plagiarism-resistant testing results.

\subsection{TSINC's Acceptance}

Although TSINC has significant features and can be used easily in various courses and training programs, there are still difficulties that prevent TSINC from being adopted quickly. The major reason is that TSINC, a simple testing method, challenges a few widely-accepted norms in teaching and testing. The major difficulties in accepting the TSINC testing method are the changes to one's comfortable conventions.

In traditional testing methods, the following norms are accepted widely:

1. Test participants should be given enough time to answer questions.

2. Test participants should be given the freedom to navigate through all the questions at any time.

3. Only those test participants who have correctly answered all or most of the questions in a test will obtain high grades.

4. Typically, test grades should not be adjusted by the instructor after the test. If the instructor adjusts grades after the test, the instructor may have made some incorrect choices designing the test. 
These norms fit the paper-based, traditional testing environment. Today, where most courses and programs are Internet-based instead of paper-based, instructors face new challenges and the promise of new opportunities. Therefore, the standard rules and patterns are no longer absolute. Some measures may change to meet new challenges and to take advantage of new opportunities. Whether these norms should change is a more complicated issue to be discussed. A few of the reasons and the consequences of these changes are:

1. Many real-world scenarios are time-sensitive; answering questions in a time-pressed environment is good training. Indeed, time-pressed tests will influence test participants' performance negatively, but such influence can be compensated through calibration. The benefit of introducing the time-pressed characteristic is the plagiarism-resistant online tests. If test participants must have enough time to consider the questions before answering questions, then TSINC tests should not be used in this course or this subject.

2. Many real-world scenarios are sequential; answering questions in a sequential test is good training. Indeed, sequential tests will negatively influence test participants' performance, but such influence can be compensated through calibration. The advantage of introducing the sequential characteristic is the plagiarism-resistant online tests.

3. Suppose the test questions are too difficult, and the time limit is short; even the honest learners correctly answer only a portion of the questions. In that case, they still deserve the high grades generated through the calibration process. Such a calibration process should be announced to test participants before the tests to reduce anxiety.

4. Instructors often feel the tests were too difficult or too easy. Instructors usually do not adjust grades unless the grade shifting is quite significant. All TSINC tests have a built-in calibration process so that test participants' performance can be evaluated accurately, and instructors do not need to feel guilty when the test he/she made is slightly harder or easier than he/she expected.

If satisfactory test results are desired, the above points should be explained to test participants so that they are not surprised when they take TSINC tests. Understandably, test participants are not familiar with time-pressed tests, sequential question answering, and post-test grade calibration. These are all new characteristics, so that detailed introductions and explanations are necessary before TSINC tests are held.

In the past, some characteristics, such as sequential question answering, seemed interesting but did not bring in many benefits; therefore, people did not have much motivation to introduce them. When a few characteristics were combined properly, a sizeable benefit was produced: a plagiarism-resistant online testing method. Now there are motivations to introduce and explain these characteristics.

\subsection{Theoretical Explorations}

In this section, I would like to briefly report some of my preliminary explorations in the TSINC testing method's theoretical side.

This method is based on two key observations:

1. Plagiarism introduces extra activities that honest test participants do not need to do. 
2. Plagiarism consumes time.

Based on the two above observations, a few basic principles were developed. These principles were used as guidelines in creating the TSINC testing method.

1. Reduce the time limit of the tests, so that time cost becomes critical.

2. Use various measures to reduce the size of the windows wherein plagiarism may happen.

3. Use various measures to increase the cost of time for plagiarism.

4. Tie the hands of both honest learners and potential plagiarists with test tasks so that they cannot commit plagiarism passively or actively.

5. The side effects of some necessary measures should be compensated properly.

Other plagiarism-resistant testing methods can also be developed if these principles are followed in other ways.

\section{TSINC Test Cases}

The TSINC testing method was a tool I devised to evaluate students' performance properly when I taught courses online during the COVID-19 pandemic. This method has been used in the exams of three courses in Fall 2020. These TSINC tests were implemented on the Moodle platform. Table 1 briefly describes these TSINC test cases. Some students, who had documented learning disability issues, were given a longer time limit (50\% longer than other students) to reduce their stress level.

Table 1: Brief Description of Some TSINC Test Cases

\begin{tabular}{|c|c|c|c|c|}
\hline \multirow{2}{*}{ Course ID } & \multirow{2}{*}{ Number of Students } & \multirow{2}{*}{ Time Limit (minutes) } & \multicolumn{2}{|c|}{ Grade Average } \\
\cline { 4 - 5 } & & & Before Calibration & After Calibration \\
\hline 1 & 87 & 20 & $51.60 \%$ & $74.90 \%$ \\
\hline 2 & 31 & 20 & $53.40 \%$ & $75.70 \%$ \\
\hline 3 & 28 & 20 & $58.60 \%$ & $76.30 \%$ \\
\hline 4 & 87 & 50 & $72.76 \%$ & $76.24 \%$ \\
\hline 5 & 31 & 30 & $66.94 \%$ & $75.94 \%$ \\
\hline 6 & 29 & 50 & $62.40 \%$ & $74.14 \%$ \\
\hline
\end{tabular}

In the first three tests, students encountered TSINC tests for the first time. I had explained that the time would be very tight; I would adjust the grade if they obtained a low mark. Checking back, I believe that my explanations were not enough. Therefore, students' performances in the first three tests were lower than that in the last three tests. They complained that there was not enough time, and they worried that their course grades would be too low. After I adjusted the grades and made detailed explanations to the students, they were much relieved. In the last three tests, students' performance was much better, and they have developed time management strategies.

Overall, the TSINC tests reached the goals I set when I developed this testing method. Students told me that these tests were fair, and plagiarism was under control as far as they 
knew. At the same time, things were quite different in some other courses. I have taught over $2 / 3$ of the students in person in other courses before the pandemic. Generally, good students in this course also have good grades in the records; the grades of average students and poor students are also consistent with previous records. Such consistency demonstrates that the grades of these courses were not interfered with by potential plagiarism when human monitoring was unavailable.

The following are some comments from students regarding the TSINC tests:

"The online tests were great for evaluating students since it discouraged cheating (due to the nature of the one-way test, plus the time restriction) and it allowed students who studied to benefit from doing so."

"Although I was very nervous about this final exam I think you did a terrific job making it very fair. I worked hard to study for it and it felt like a good test of my knowledge."

"Good Exam! I found it fair, and no time to do anything else but read the questions and answer. I think I did OK."

Apparently, if there were plagiarists, they would not feel happy about the TSINC testing method, but they cannot openly oppose the method.

The size of the presented TSINC test cases is small. Due to the current pandemic situation, such a method is needed and might be useful for many instructors. Therefore, I present this method in its early form to be criticized, improved, and used to combat plagiarism in various universities and schools during this pandemic time and in the future.

\section{Conclusions}

The TSINC testing method can be used to evaluate student's learning in various courses and training programs in an online environment. Its major feature is that it can impact plagiarism negatively without in-person monitoring. In other words, the TSINC testing method has a built-in plagiarism-resistant ability.

The TSINC testing method was based on two key observations: (1) plagiarism introduces extra activities that honest test participants do not need to do; (2) plagiarism consumes time.

In TSINC tests, a few widely-accepted conventions were replaced by new ones. TSINC tests can only be organized successfully when the proposed changes are explained clearly to test participants.

The TSINC testing method has been used in university teaching, and the results were very promising. This method is still a new one; more test cases and analyses are needed. Suggestions and critics are welcome.

\section{Acknowledgment}

This work would not have been possible without the atmosphere of academic freedom at the University of Prince Edward Island and the support from my colleagues and the administration in the School of Mathematical and Computational Sciences at the university. 


\author{
Affiliation: \\ Yingwei Wang \\ School of Mathematical and Computational Sciences \\ University of Prince Edward Island \\ Charlottetown, Canada \\ E-mail: ywang@upei.ca \\ URL: http://smcs.upei.ca/ `ywang/
}

SocArXiv Website SocArXiv Preprints

Preprint

doi.org/10.31235/osf.io/u4zcs https://socopen.org/

https://osf.io/preprints/socarxiv

Submitted: January 22, 2021

Accepted: January 23, 2021 